\title{
Research on Dynamic-State Optimized Operation of Natural Gas Pipeline Network
}

\author{
Yi Yang ${ }^{1, ~ a ~}$, Lu Zhang ${ }^{2, b}$ Enbin Liu ${ }^{2, c}$ and Jing Tang ${ }^{3, d}$ \\ ${ }^{1}$ Beijing Oil and Gas Pipeline Control Center, Beijing, China \\ ${ }^{2}$ Southwest Petroleum University, Chengdu, China \\ ${ }^{3}$ PetroChina Southwest Oil and Gas Field Company, Chengdu, China \\ ayangyi.xn@petrochina.com.cn, ${ }^{\mathrm{b}}$ 13648010939@163.com, ${ }^{\mathrm{s}}$ sunriselebpsb@163.com,
}

Keywords: pipeline network, dynamic, optimal operation, mathematical model, linearized algorithm. Abstract. Natural gas is usually on unsteady-state condition, the change of volumes and pressures will bring different economic benefit at different time, so that the operation of natural gas pipeline should be optimized to guarantee the safety. By analyzing the pressure and volume varying with time, scheme could be made to increase the integrity of the system while minimizing cost. This paper develops a mathematic model to simulate the dynamic state of the system, considering maximum profit as the object function and the flow, pressure, dynamic flow equation of nodes as constraints. This paper adopts improved linearized algorithms to solve it. The result optimizes network operation.

\section{Introduction}

The major task natural gas pipeline manager faced with is to ensure the system operate economically and reasonably. A good operation scheme can guarantee safety and satisfy users' needs. Besides, the purpose of saving costs should also be concluded. So it is necessary to optimize natural gas pipeline network. When natural gas operate at steady state, it is a highly effective method to use steady-state optimized model to analyze and optimize the network ${ }^{[1,2]}$. But natural gas is usually on unsteady-state condition in pipeline in practice production, the change of natural volumes and pressures of gas supply and users will lead to different economic benefits and pipeline flow ${ }^{[3,4,5]}$. This situation calls for a research about dynamic-state optimized operation of natural gas pipeline network.

\section{Dynamic-state optimized operation model of natural gas pipeline network}

Temperature change is beyond consideration, which means gas flow is regarded as isothermal flow. Object function. The gas sales revenue can be the object of the model:

$$
\max F=\int_{0}^{T} \sum_{i=1}^{N_{n}} S_{i} Q_{i} d t
$$

In Eq.(1), $Q_{i}(\mathrm{~m} 3 / \mathrm{d})$ is the intake(distribute) flow of node $i$, it is positive when node $i$ distribute gas and negative when intake flow, otherwise, the value of $Q_{i}$ is $0 . S_{i}$ (yuan $/ \mathrm{m}^{3}$ ) is the cost coefficient about purchase/sale gas. $N_{n}$ is the total number of nodes. T is time period and t means at any time.

Constraints. Users have different demands of gas volumes at different times, and production unit produces different amount of natural gas at different times due to reality. This situation leads to time differences of intake(distribute) flow of network system and different constraints at different times:

$$
Q^{j}{ }_{i \min } \leq Q^{j}{ }_{i} \leq Q^{j}{ }_{i \max } \quad\left(i=1,2, \mathrm{~L} N_{n}\right)
$$

In Eq.(2), $Q^{j}{ }_{i}(\mathrm{~m} 3 / \mathrm{d})$ means the intake(distribute) flow of node i at time $\mathrm{j}, Q^{j}{ }_{i \min }$ and $Q^{j}{ }_{i \max }$ means the minimum and maximum intake(distribute) flow of node $i$ at time $j$ respectively.

The change of node pressure is also time-dependent. So the Pressure constraint is:

$$
P^{j}{ }_{i \min } \leq P^{j}{ }_{i} \leq P^{j}{ }_{i \max } \quad\left(i=1,2, \mathrm{~L} N_{n}\right)
$$


In Eq.(3), $P^{j}{ }_{i}(\mathrm{~Pa})$ means the pressure of node $\mathrm{i}$ at time $\mathrm{j}, P^{j}{ }_{i \min }$ and $P^{j}{ }_{i \max }$ means the minimum and maximum allowable pressure of node $\mathrm{i}$ at time $\mathrm{j}$ respectively.

If the total number of pipeline is $N_{p}$, then the gas pressure $P_{k}$ must smaller than the MAOP:

$$
P_{k} \leq P_{k \max } \quad\left(k=1,2, \mathrm{~L}, N_{p}\right)
$$

In Eq.(4), $P_{k}(\mathrm{~Pa})$ means the pressure in pipeline $\mathrm{k}$, and $P_{k \max }$ means the MAOP of pipeline k.

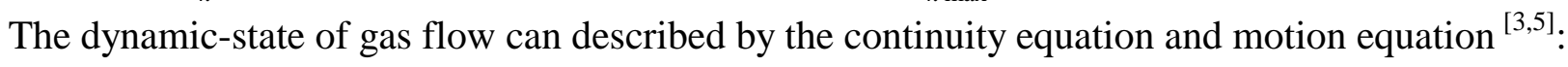

$$
\begin{aligned}
& A \frac{\partial \rho}{\partial t}+\frac{\partial M}{\partial x}=0 \\
& \frac{\partial M}{\partial t}+\frac{1}{A} \frac{\partial}{\partial x}\left(A^{2} P+\frac{M^{2}}{\rho}\right)+A g \rho \sin \theta+\frac{\lambda}{2} \frac{M^{2}}{D A \rho}=0
\end{aligned}
$$

$M(\mathrm{~kg} / \mathrm{s})$ means the mass flow rate, $A\left(\mathrm{~m}^{2}\right)$ means the flow area, gas density is $\rho\left(\mathrm{kg} / \mathrm{m}^{3}\right)$, time is $t(\mathrm{~s})$, pipeline length is $x(\mathrm{~m})$ and $P(\mathrm{~Pa})$ is gas pressure, $\theta(\mathrm{rad})$ is the angle between pipeline and horizontal plane, $\mathrm{D}(\mathrm{m})$ is the inner diameter of pipe, and $\lambda$ is the hydraulic coefficient of the pipeline.

The flow equilibrium equations for pipeline network with Nn nodes can be wrote as follows: ${ }^{[2]}$

$$
\sum_{\substack{k \in C_{i} \\ i=1}}^{N n} \alpha_{i k} M_{i k}+Q_{i}=0
$$

In Eq.(7), $C_{i}$ means the set of units connect to node $i$, and $M_{i k}(\mathrm{~kg} / \mathrm{s})$ is the absolute value of inflows or outflows at unit k connected to node $i$ at one point, $Q_{i}(\mathrm{~kg} / \mathrm{s})$ is the flow exchange between node $i$ and outside, it is positive when flow inside and negative when outside, $a_{i k}$ is the coefficient, its value is +1 when gas flow into node $i,-1$ when flow out, otherwise, the value of $a_{i k}$ is 0 .

After analyzing different time interval and space dimension, optimization variables are:

$$
X=\left[Q_{i}^{k}, P_{i}^{k}, M_{i}^{k}\right](i=1,2,3 \mathrm{~L} \quad N ; k=1,2,3 \mathrm{~L} n)
$$

In Eq.(8), $\mathrm{N}$ means the total number of nodes, and $\mathrm{n}$ is the total number of time interval.

\section{Calculation example}

A pipeline network is shown in Fig. [7] $^{[7]}$ The parameters are shown in Table.1. The pressure constraint is $0.5 \mathrm{MPa} 4.5 \mathrm{MPa}$. After dividing the model into 12 time intervals, this paper use an improved linearized algorithm ${ }^{[8]}$ to solve it. Gas price and constraints of node 1 to node 8 are listed in Table 2. The optimization goal is to maximum benefit. Table 3 lists optimized results and benefits.

Table 1 Pipeline Data

\begin{tabular}{|c|c|c|c|c|c|c|c|c|c|c|c|}
\hline Pipeline & 1 & 2 & 3 & 4 & 5 & 6 & 7 & 8 & 9 & 10 & 11 \\
\hline Up node & 13 & 1 & 2 & 2 & 4 & 4 & 1 & 1 & 6 & 6 & 7 \\
\hline Down node & 12 & 2 & 4 & 3 & 3 & 5 & 6 & 7 & 7 & 5 & 8 \\
\hline Length(km) & 8.05 & 16.09 & 6.44 & 9.66 & 8.05 & 8.05 & 9.66 & 11.27 & 7.24 & 8.05 & 10.70 \\
\hline Diameter(mm) & 406.4 & 304.8 & 304.8 & 304.8 & 254.0 & 254.0 & 355.6 & 406.4 & 304.8 & 254.0 & 254.0 \\
\hline Pipeline & 12 & 13 & 14 & 15 & 16 & 17 & 18 & 19 & 20 & 21 & 22 \\
\hline Up node & 5 & 5 & 3 & 11 & 9 & 10 & 8 & 14 & 8 & 12 & 11 \\
\hline Down node & 9 & 10 & 11 & 10 & 8 & 9 & 11 & 15 & 14 & 1 & 16 \\
\hline Length(km) & 14.48 & 14.48 & 19.31 & 9.66 & 12.87 & 11.27 & 17.70 & 4.83 & 2.00 & 2.00 & 2.00 \\
\hline Diameter(mm) & 254.0 & 254.0 & 254.0 & 203.2 & 254.0 & 203.2 & 203.2 & 254.0 & 554.0 & 554.0 & 554.0 \\
\hline
\end{tabular}




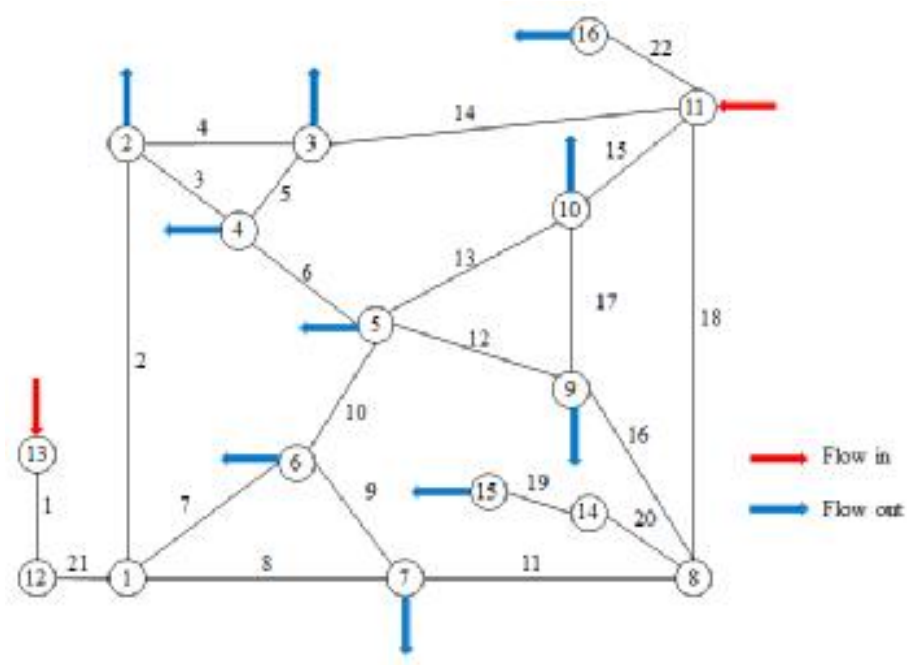

Fig. 1 Schematic diagram of natural pipeline network

Table 2 Node data(flow rate: $10^{4} \mathrm{~m}^{3} / \mathrm{d}$ )

\begin{tabular}{|c|c|c|c|c|c|c|c|c|c|c|c|c|c|c|}
\hline \multirow{2}{*}{ Node } & \multirow{2}{*}{$\begin{array}{c}\text { Price } \\
\left(\text { yuan } / \mathrm{m}^{3}\right)\end{array}$} & \multirow{2}{*}{ Boundary } & \multicolumn{12}{|c|}{ Time interval } \\
\hline & & & 1 & 2 & 3 & 4 & 5 & 6 & 7 & 8 & 9 & 10 & 11 & 12 \\
\hline \multirow{2}{*}{1} & \multirow{2}{*}{0.00} & Max & 0 & 0 & 0 & 0 & 0 & 0 & 0 & 0 & 0 & 0 & 0 & 0 \\
\hline & & Min & 0 & 0 & 0 & 0 & 0 & 0 & 0 & 0 & 0 & 0 & 0 & 0 \\
\hline \multirow{2}{*}{2} & \multirow{2}{*}{1.00} & Max & 70 & 55 & 55 & 80 & 65 & 55 & 85 & 55 & 40 & 30 & 30 & 40 \\
\hline & & Min & 60 & 45 & 45 & 70 & 55 & 45 & 75 & 45 & 30 & 20 & 20 & 30 \\
\hline \multirow{2}{*}{3} & \multirow{2}{*}{1.10} & Max & 55 & 40 & 40 & 65 & 50 & 40 & 70 & 40 & 25 & 15 & 15 & 25 \\
\hline & & Min & 45 & 30 & 30 & 55 & 40 & 30 & 60 & 30 & 15 & 5 & 5 & 15 \\
\hline \multirow{2}{*}{4} & \multirow{2}{*}{0.98} & Max & 55 & 55 & 55 & 55 & 55 & 55 & 55 & 55 & 55 & 55 & 55 & 55 \\
\hline & & Min & 45 & 45 & 45 & 45 & 45 & 45 & 45 & 45 & 45 & 45 & 45 & 45 \\
\hline \multirow{2}{*}{5} & \multirow{2}{*}{0.95} & Max & 70 & 55 & 55 & 40 & 30 & 30 & 40 & 55 & 80 & 65 & 55 & 85 \\
\hline & & Min & 60 & 45 & 45 & 30 & 20 & 20 & 30 & 45 & 70 & 55 & 45 & 75 \\
\hline \multirow{2}{*}{6} & \multirow{2}{*}{1.13} & Max & 40 & 40 & 40 & 40 & 40 & 40 & 40 & 40 & 40 & 40 & 40 & 40 \\
\hline & & Min & 30 & 30 & 30 & 30 & 30 & 30 & 30 & 30 & 30 & 30 & 30 & 30 \\
\hline \multirow{2}{*}{7} & \multirow{2}{*}{1.15} & Max & 40 & 40 & 65 & 55 & 40 & 25 & 15 & 15 & 25 & 50 & 40 & 70 \\
\hline & & Min & 30 & 30 & 55 & 45 & 30 & 15 & 5 & 5 & 15 & 40 & 30 & 60 \\
\hline \multirow{2}{*}{8} & \multirow{2}{*}{0.00} & Max & 0 & 0 & 0 & 0 & 0 & 0 & 0 & 0 & 0 & 0 & 0 & 0 \\
\hline & & Min & 0 & 0 & 0 & 0 & 0 & 0 & 0 & 0 & 0 & 0 & 0 & 0 \\
\hline
\end{tabular}

Table 3 Optimized flow rate $\left(10^{4} \mathrm{~m}^{3} / \mathrm{d}\right)$, pressure $(\mathrm{MPa})$ and benefits on every time interval

\begin{tabular}{|c|c|c|c|c|c|c|c|c|c|c|}
\hline \multicolumn{2}{|c|}{ Time interval } & Node1 & Node2 & Node3 & Node4 & Node 5 & Node6 & Node7 & Node8 & $\begin{array}{c}\text { Benefits } \\
\left(10^{4} \text { yuan/d) }\right.\end{array}$ \\
\hline \multirow{2}{*}{1} & Flow rate & 0.00 & 70.00 & 55.00 & 55.00 & 70.00 & 40.00 & 40.00 & 0.00 & \multirow{2}{*}{11.1} \\
\hline & Pressure & 3.483 & 1.609 & 1.380 & 1.464 & 1.473 & 3.235 & 3.244 & 1.480 & \\
\hline \multirow{2}{*}{2} & Flow rate & 0.00 & 55.00 & 40.00 & 55.00 & 55.00 & 40.00 & 40.00 & 0.00 & \multirow{2}{*}{9.9} \\
\hline & Pressure & 3.371 & 1.890 & 1.722 & 1.774 & 1.779 & 3.148 & 3.155 & 1.609 & \\
\hline \multirow{2}{*}{3} & Flow rate & 0.00 & 55.00 & 40.00 & 55.00 & 55.00 & 40.00 & 65.00 & 0.00 & \multirow{2}{*}{10.6} \\
\hline & Pressure & 3.391 & 1.900 & 1.731 & 1.783 & 1.787 & 3.144 & 3.146 & 1.609 & \\
\hline \multirow{2}{*}{4} & Flow rate & 0.00 & 80.00 & 65.00 & 55.00 & 40.00 & 40.00 & 55.00 & 0.00 & \multirow{2}{*}{11.6} \\
\hline & Pressure & 3.470 & 1.637 & 1.402 & 1.544 & 1.641 & 3.217 & 3.221 & 1.513 & \\
\hline \multirow{2}{*}{5} & Flow rate & 0.00 & 65.00 & 50.00 & 55.00 & 30.00 & 40.00 & 40.00 & 0.00 & \multirow{2}{*}{10.0} \\
\hline & Pressure & 3.280 & 1.844 & 1.680 & 1.768 & 1.836 & 3.067 & 3.073 & 1.609 & \\
\hline \multirow[t]{2}{*}{6} & Flow rate & 0.00 & 55.00 & 40.00 & 55.00 & 30.00 & 40.00 & 25.00 & 0.00 & \multirow{2}{*}{8.9} \\
\hline & Pressure & 2.709 & 1.441 & 1.314 & 1.370 & 1.411 & 2.513 & 2.522 & 1.208 & \\
\hline
\end{tabular}


Table 3 Cont.

\begin{tabular}{|c|c|c|c|c|c|c|c|c|c|c|}
\hline \multirow{2}{*}{7} & Flow rate & 0.00 & 85.00 & 70.00 & 55.00 & 40.00 & 40.00 & 15.00 & 0.00 & \multirow{2}{*}{10.9} \\
\cline { 2 - 12 } & Pressure & 3.545 & 1.645 & 1.397 & 1.556 & 1.675 & 3.323 & 3.338 & 1.555 & \\
\hline \multirow{2}{*}{8} & Flow rate & 0.00 & 55.00 & 40.00 & 55.00 & 55.00 & 40.00 & 15.00 & 0.00 & \multirow{2}{*}{9.2} \\
\cline { 2 - 11 } & Pressure & 3.350 & 1.872 & 1.704 & 1.758 & 1.764 & 3.148 & 3.161 & 1.599 & \\
\hline \multirow{2}{*}{9} & Flow rate & 0.00 & 40.00 & 25.00 & 55.00 & 80.00 & 40.00 & 25.00 & 0.00 & \multirow{2}{*}{9.0} \\
\cline { 2 - 12 } & Pressure & 3.159 & 1.643 & 1.470 & 1.483 & 1.458 & 2.936 & 2.948 & 1.343 & \\
\hline \multirow{2}{*}{10} & Flow rate & 0.00 & 30.00 & 15.00 & 55.00 & 65.00 & 40.00 & 50.00 & 0.00 & \multirow{2}{*}{8.7} \\
\cline { 2 - 11 } & Pressure & 3.138 & 1.928 & 1.786 & 1.791 & 1.769 & 2.925 & 2.929 & 1.594 & \multirow{2}{*}{8.1} \\
\hline \multirow{2}{*}{11} & Flow rate & 0.00 & 30.00 & 15.00 & 55.00 & 55.00 & 40.00 & 40.00 & 0.00 & \multirow{2}{*}{10.5} \\
\cline { 2 - 11 } & Pressure & 3.042 & 1.910 & 1.780 & 1.787 & 1.779 & 2.846 & 2.851 & 1.590 & 0.00 \\
\hline \multirow{2}{*}{12} & Flow rate & 0.00 & 40.00 & 25.00 & 55.00 & 85.00 & 40.00 & 70.00 & 1.478 & \\
\cline { 2 - 10 } & Pressure & 3.381 & 1.814 & 1.624 & 1.636 & 1.596 & 3.116 & 3.117 & \\
\hline
\end{tabular}

\section{Conclusion}

1、Ignore the temperature change, this paper build a dynamic-state optimized operation model on the basis of analyzing dynamic simulation model of pipeline network;

2、The features of this dynamic optimized model are as follows: multivariable, non-linear, non-convex feasible region composed by constraints, equality and inequality constraint equations;

3、 This paper adopts improved linearized algorithm to solve the model of an example. The result shows that it can solve the model built in this paper quickly and effectively.

\section{Acknowledgements}

This work was financially supported by the CNPC technical research and applied research of optimization on large-scale natural gas pipeline network (2013B-3410).

\section{References}

[1] Y. Yang, C. J. Li, "Study on Steady-State Optimized Operation of Natural Gas Pipeline Network", Journal of Oil and Gas Technology, vol.27, pp.278-280, 2005.

[2] Y. Yang, C. J. Li. S. Y. Shang, "Study on Gas Transmission and Distribution Optimization of Pipeline System”, Natural Gas Industry. Vol.26, pp.123-125,2006.

[3] A. J.Osiadacz. "Dynamic Optimization of High Pressure Gas network", PSIG 26th PSIG Annual Meeting, 1994.

[4] H. H. Rachford, R. G.. Carter. “Optimizing Pipeline Control In Transient Gas Flow”, PSIG 32th PSIG Annual Meeting, 2000.

[5] D. R. Rusnak, E. Philip, "Ferber. Automated Pipeline Optimization(APO) for Nominations Management \& Compressor Fuel Minimization”, PSIG 36th PSIG Annual Meeting 2004

[6] C. J. Li, Pipeline Transmission of Natural Gas. Petroleum Industry Press, 2000.

[7] C. J. Li, Z. Q. Zeng, M. Z. Jiang, “Analysis on Unsteady Flow in Natural Gas Pipeline System”, Natural Gas Industry, vol. 14, pp. 54-59, 1994.

[8] Y. Yang, "Research on Optimized Operation of Natural Gas Pipeline Network and Optimization Method", PhD thesis, Southwest Petroleum University, Chengdu, ON, China, 2006. 
[9] E. B. Liu, C. J. Li, L. T. Yang, D. Wang, "Research on the Optimal Energy Consumption of Oil Pipeline", Journal of Environmental Biology, vol.36, pp. 703-711, 2015.

[10] E. B. Liu, C. J. Li, Y. Yang, "Optimal Energy Consumption Analysis of Natural Gas Pipeline”, Scientific World Journal, vol.4, pp. 57-78, 2014. 